\title{
Total MSAS Score
}

National Cancer Institute

\section{Source}

National Cancer Institute. Total MSAS Score. NCI Thesaurus. Code C125365.

A subscale of the Memorial Symptom Assessment Scale that is the average of the symptom scores of all 32 symptoms in the full MSAS instrument. 